\title{
INTEGRATED KNOWLEDGE-BASED TOOLS FOR DOCUMENTING AND MONITORING DAMAGES TO BUILT HERITAGE
}

\author{
R. Cacciotti ${ }^{\mathrm{a}, *}$ \\ ${ }^{a}$ Institute of Theoretical and Applied Mechanics of the Academy of Sciences of the Czech Republic, Prosecká 76, 190 \\ 00, Praha 9, Czech Republic - cacciotti@itam.cas.cz
}

Commission VI, WG VI/4

KEY WORDS: Built heritage, Ontology, Knowledge-based system, Documentation, Damage

\begin{abstract}
:
The advancements of information technologies as applied to the most diverse fields of science define a breakthrough in the accessibility and processing of data for both expert and non-expert users. Nowadays it is possible to evidence an increasingly relevant research effort in the context of those domains, such as that of cultural heritage protection, in which knowledge mapping and sharing constitute critical prerequisites for accomplishing complex professional tasks. The aim of this paper is to outline the main results and outputs of the MONDIS research project. This project focusses on the development of integrated knowledge-based tools grounded on an ontological representation of the field of heritage conservation. The scope is to overcome the limitations of earlier databases by the application of modern semantic technologies able to integrate, organize and process useful information concerning damages to built heritage objects. In particular MONDIS addresses the need for supporting a diverse range of stakeholders (e.g. administrators, owners and professionals) in the documentation and monitoring of damages to historical constructions and in finding related remedies. The paper concentrates on the presentation of the following integrated knowledgebased components developed within the project: (I) MONDIS mobile application (plus desktop version), (II) MONDIS record explorer, (III) Ontomind profiles, (IV) knowledge matrix and (V) terminology editor. An example of practical application of the MONDIS integrated system is also provided and finally discussed.
\end{abstract}

\section{INTRODUCTION}

In the last 10 years a huge amount of resources has been employed in the digitalization of cultural heritage, in an effort to integrate and standardize the available information into a formal management platform. Past research projects mainly focused on the following areas of $\mathrm{CH}$ documentation: (i) digital libraries, (ii) digital preservation systems capable of supporting long-term access to multi-sourced, multi-formatted and distributed content from a broad range of application domains; (iii) long-term usability of scientific results; (iv) web archiving; (v) digitization technologies; (vi) digital cultural experiences such as remote visits of museums and sites; (vii) virtual heritage systems, capable of capturing, rendering, modelling and visualizing cultural artefacts; (viii) retrieval and use information systems, enhancing information and knowledge retrieval through efficient search engines able to deliver results from complex multimedia resources, from distributed collections and in a multilingual environment.

The cultural heritage domain is indeed a peculiar and challenging one. By its definition cultural heritage encompasses a wide range of objects, divided into several main categories: tangible cultural heritage including movable (paintings, sculptures, coins, manuscripts) immovable (monuments, archaeological sites, and so on) and underwater heritage (shipwrecks, underwater ruins and cities); intangible cultural heritage comprising oral traditions, performing arts, rituals field; natural heritage individuating natural sites with cultural aspects such as cultural landscapes, physical, biological or geological formations (Jokilehto, 1990). The resulting heterogeneity of information types involves multi-layered sets of data dealing with the identification and description of various aspects related to cultural heritage: for example data sets concerning the historical background of $\mathrm{CH}$ objects, their physical conditions, the structural, architectonic and artistic characteristics or the possible interventions that can be carried out in a specific context. The complexity of data, often produced by exploiting a variety of methods and techniques, is further affected by an interpretative process depending on the subjective perspective of reporters (e.g. engineers, architects, archaeologists, historians, restorers, administrators, etc.), which makes the cultural heritage domain even an harsher environment to control.

Although a number of integrated tools for assisting users in the management of cultural heritage information have been developed in the past - for example the CIDOC-CRM ontology (Doerr, 2003) intended to facilitate the integration, mediation, and interchange of heterogeneous cultural heritage information; the BRICKS (Meghini et Al., 2005) and CALIMERA (Faletar, 2005) projects dedicated to the networking of the main stakeholders involved in cultural heritage administration for the purpose of digitalization of assets related data, or the DigiCULT (EC DG Information Society, 2002), CASPAR (European Commission, 2006) and CHARISMA (European Communities, 2009) projects with the objective to improve the access to databases exploiting digitalization of data and their harmonization - considerable effort is still required in delivering

\footnotetext{
* Corresponding author. Tel: +420 286885320; fax: +420 286884634. E-mail address: cacciotti@itam.cas.cz (R. Cacciotti)
} 
truly semantically empowered and friendly to use tools (Konstantinos et Al., 2012).

As evidenced by many studies, in order to better address the proper applicability of Semantic Web technologies to the domain of cultural heritage protection, research needs to be focused mainly on the following points: understanding the multidisciplinary nature of analytical data available in the field in the perspective of optimal data integration and knowledge reuse; accommodating as much as possible flexible and personalized semantics for the expression of subjective opinions in the ontological framework, considering at the same time the limitations set by ontology engineering principles; validating the ontology by fostering the participation of end-users in its development, so to avoid syntactic defects, unintended redundancy, modelling and conceptual defects, semantic defects and inconsistencies; addressing the accessibility of information and tools developed, supporting a multilingual approach to knowledge representation, access and translation; implementing automatic and user-friendly interfaces necessary to maximize the involvement of the cultural heritage community; finally, addressing, resolving, and incorporating into the semantic web tools ownership, permission of use, trust, and copyrights issues which are very relevant in the perspective of the project sustainability.

In this context the MONDIS project attempts to encompass all these issues, concentrating on the development of an ontological framework able to coordinate an automated reasoning behind the documentation of damages to built heritage, their diagnosis and possible interventions.

This paper outlines the main results of the MONDIS project and in particular the knowledge-based tools developed in order to document and monitor damages to built heritage. The document is divided into the following parts: section 2 presents the MONDIS system and its integrated knowledge-based applications including the MONDIS mobile/desktop, the MONDIS explorer, the Ontomind profile, the knowledge matrix and the terminology editor; section 3 provides a record example in order to endorse a more comprehensive understanding of the system functionalities and workflow; finally section 4 concludes on the main results of the MONDIS project discussing the advantages and limitations of the system as well as its possible developments in future work.

\section{MONDIS SYSTEM}

MONDIS is a damage-centered, knowledge-based information system concerning immovable cultural heritage objects. The correct performance of its functionalities, such as the semantic reasoning and advanced searching, is ensured by the exploitation of an ontology called Monument Damage Ontology, described in details in previously published papers (Blaško et Al., 2012) and (Cacciotti et Al., 2013).

The system features a number of complementary software tools which enable users to gather, edit, integrate and process data. More specifically MONDIS includes inputting applications, such as the mobile/desktop app and the Ontomind profile, as well as visualizing or supporting tools, namely the MONDIS explorer, the knowledge matrix and the terminology editor.

As shown in Figure 1, the integration of the developed knowledge-based tools follows a clear pattern of dataflow: building inspection and damage information is gathered directly on-site by using the MONDIS mobile software (or its desktop emulation); once the report is complete, data are uploaded to the server, where a validation process is triggered in order to verify the quality of the record and avoid unusable knowledge. At this point data flow into the record explorer, becoming available to the public users for browsing; once shown in the MONDIS explorer application, records can be integrated with further details which are not collected directly on-site, via Ontomind profiles (e.g. structural evolution; laboratory testing, diagnosis and possible remedies etc.). The entries available in the explorer, in particular damage identification and description data sets, are semantically linked by the exploitation of the monument damage ontological model to their diagnosis and possible remedies which can be visualized by users in the knowledge matrix application. All tools are supported by backing data, such as definitions, images and synonyms, provided in the terminology editor. The applications discussed above are presented and thoroughly described in the following paragraphs.

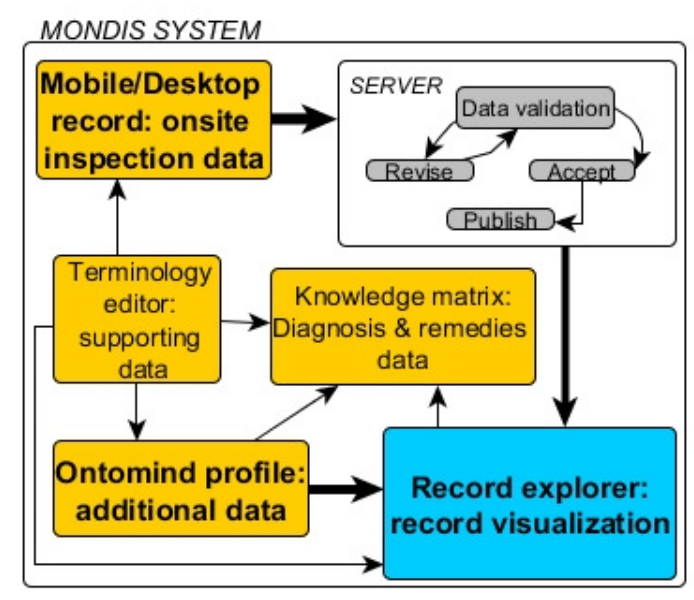

Figure 1. MONDIS system: dataflow

\subsection{MONDIS mobile/desktop application}

The MONDIS mobile (Fig.2) is a multi-platform application intended for portable electronic devices such as tablets and mobile phones. Its main goal is to support professionals during the on-site inspection of buildings by providing a simple interactive tool in which relevant data concerning damages (e.g. description, measurements, and photographs) can be easily recorded. The application interface is composed of five main screens or sections, namely the list of records, the building identification, the building localization, the building inspection and the detailed assessment screens. The first screen provides users with a list of records which have been stored on the device allowing viewing their content, editing or updating an existing entry and uploading the record to the server. The building identification screen allows identifying the object by: its name/denomination (e.g. Colosseum); its morphological type (e.g. arena); its structural type (e.g. load bearing wall) and functional types (e.g. archeological site), style (e.g. Roman style). The second screen permits locating the investigated object by different means: automatically uploading the coordinates via the GPS support buit-in the device, by inserting manually the address or the cadastral reference data, by picking manually a point on the map. The building inspection screen offers the list of building components that should be inspected (e.g. roof, floor, vertical elements and foundations). Such collapsible list is structured in a hierarchical manner so to have a tree of components starting from large scale building parts at the top (e.g. roof) and ending by small scale elements at the bottom (e.g. sub-components of roof such as truss, purlin, ridge etc.). Each building component can be assigned, by means of selecting the right term from dropdown menus, with the 
corresponding materials and the visible damages. The default structure provided in the building inspection screen is one to one, meaning that there is one type of building component which can be assigned with one type of material and one type of damage. However users can customize the given tree by duplicating building components, materials and damages as needed. Finally, the detailed assessment screen allows supplementing the record with additional data available on-site. Each node of the tree structure activated in the previous screen (i.e. each selected building component, material and damage) can be integrated with media files (voice, video, picture) as well as qualitative or quantitative measurements taken during the inspection such as geometrical properties of components (e.g. wall thickness, beam length), material properties (e.g. brick water absorption) or damage properties (e.g. crack length, detachment area).

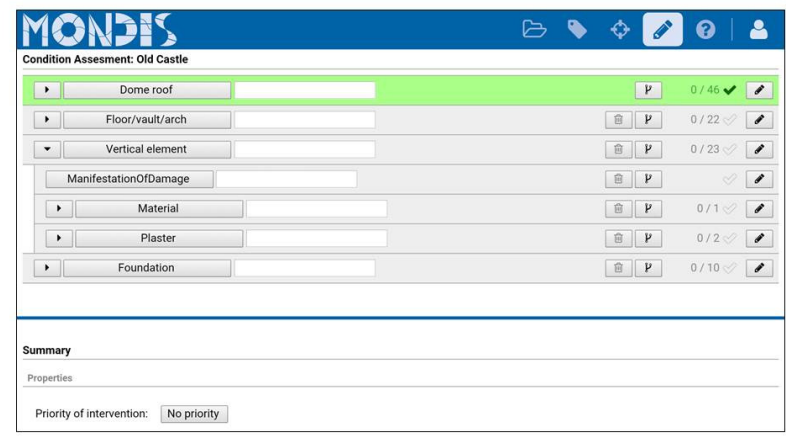

Figure 2. MONDIS mobile/desktop application

All data used by the application, like maps and terminology data, can be downloaded into the devices from the server for offline use.

Once the inspection is concluded, the record can be synchronized with the MONDIS server (WI-FI connection required) which stores the information. After the record is reviewed and accepted, as shown in Figure 1, it finally flows into the MONDIS record explorer.

The MONDIS desktop is a web-based application which emulates the mobile one and it has a similar interface. MONDIS desktop can be accessed by users via the MONDIS explorer (section 2.2) and it can be used whenever an on-site inspection has been carried out without employing electronic devices or in the case existing reports need to be digitalized into the MONDIS format.

The main advantage of MONDIS mobile/desktop compared to similar information systems is that each record is ontologically mapped enabling thus automatic data reasoning and processing necessary for advanced searching and data integration purposes.

\subsection{MONDIS explorer}

The MONDIS explorer represents the main interface in which users can search, rank and visualize the records stored by using the mobile/desktop application. The explorer is composed of two main sections: one dedicated to record retrieval and the other one involving the visualization of data contained in each record. The first section (Fig.3) enables browsing existing records by using keywords or other relevant searching parameters (e.g. damage and construction types). The result of the search can be further refined by ranking the records found according to predefined criteria such as a chronological ordering, priority of intervention or historic value. As mentioned in the previous paragraph, this section of the
MONDIS explorer can be used also to create a new inspection record by accessing the MONDIS desktop application. The outcome of the search and ranking is an ordered list of boxes containing the records which can be then visualized in detail by clicking on the view button. This action opens the record in the visualizing section of the interface described below.

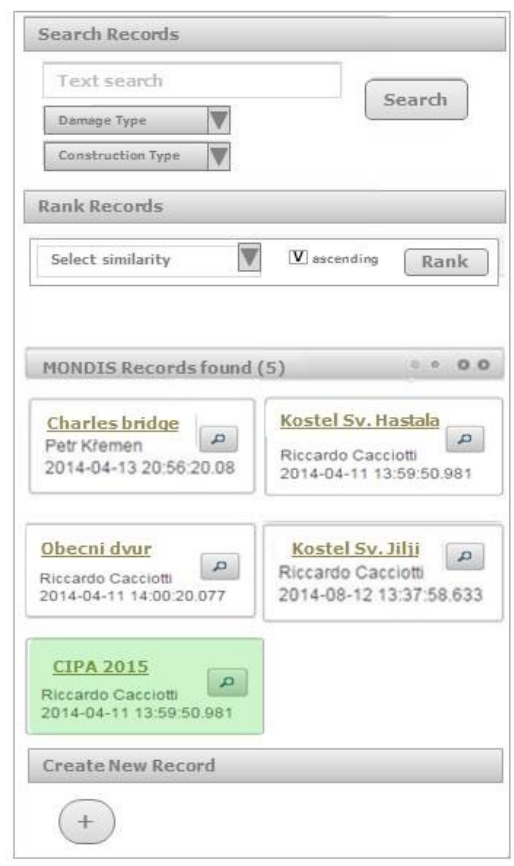

Figure 3. MONDIS explorer: record retrieval section

Records are shown into a simple table-like structure (Fig.4) including a name and localization section, a damage overview table, a MONDIS report section and finally a 'other data' table.

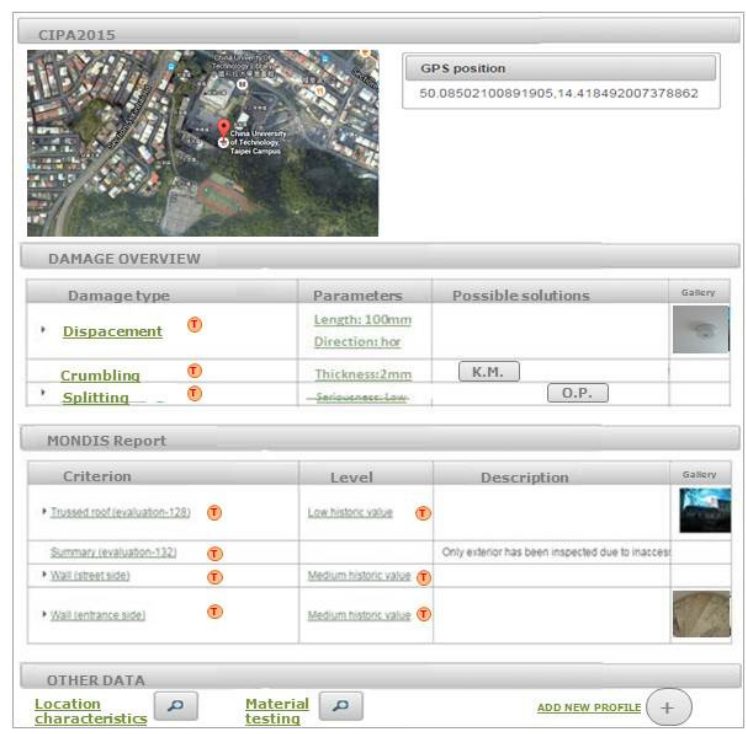

Figure 4. MONDIS explorer: record visualization section

At the top of the interface the name of the record, its location on the map and the GPS coordinates are presented. The damage overview section offers a summary of all damages present in the record, highlighting the most important parameters assessed during the inspection. Possible remedies to such damages, if 
available in the system, are shown in the 'possible solutions' column: here the 'K.M.' and 'O.P.' buttons (respectively knowledge matrix and Ontomind profile) redirect to the relevant entries in the other applications (see sections 2.3 and 2.4) dealing with the diagnosis and intervention of a particular damage. The MONDIS report section presents the full report done via MONDIS mobile/desktop, including all data gathered during the building inspection such as the description of building components, materials and damages, their measurements and photographic evidence. The 'other data' section of the explorer presents additional information integrated via Ontomind profiles (section 2.3) which might concern different aspects of the object under analysis such as its structural evolution, the surrounding characteristics or risk assessment of specific building components. In fact, being the MONDIS mobile record related to damage identification only, in the MONDIS explorer interface registered reporters can integrate their records with additional data by using the 'add new profile' button which redirects to the Ontomind application.

It should be noted that the orange circular icon on the righthand side of terms redirect to the terminology editor entry (section 2.5) in order to provide basic information such as definitions, synonyms, translations and pictures of the terminology used.

\subsection{Ontomind profile}

Ontomind (Fig.5) is a web application which visualizes the ontological mapping of records as a simple tree-like structure. In such a way data gathered during the inspection using the mobile/desktop application are mapped according to the Monument Damage Ontology, allowing the exploitation of the formalized semantic links in order to expand the record with further information.

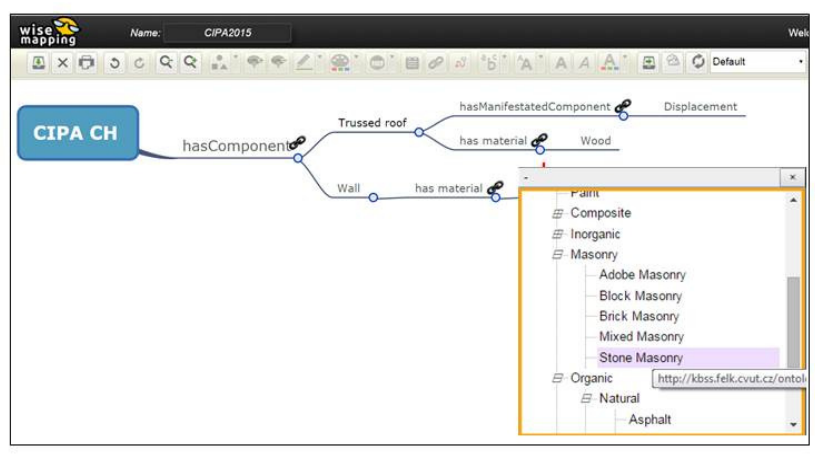

Figure 5. Ontomind profile

An Ontomind profile employs only some predefined links within the model so to individuate thematic patterns or schemes which can be easily used by users to add a series of relevant information to the record. Thematic Ontomind profiles include: object description, object evolution, location characteristics, material properties, diagnosis and intervention and risk profiles. Users can select in the MONDIS explorer from a list of readymade profiles the ones which are required. As depicted in Figure 5, once a profile is selected the user interacts with the application by means of dropdown menus at each node which provide a list of concepts or links available so to follow the predefined pattern, building in such a way the tree-like structure of data. The compiled information is then 'attached' to the relevant record the user is editing and shown in the 'other data' section in the MONDIS explorer application.

\subsection{Knowledge matrix}

The knowledge matrix is a web-based application which visualizes the knowledge concerning the diagnosis of reported damages and the possible solutions. The scope is to support non-expert users in the decision making process by sharing basic information related to the techniques and methods required to appropriately perform an intervention, including remarks on possible advantages and disadvantages of its applicability. This tool is grounded on the diagnostic part of the Monument Damage Ontology (Cacciotti et Al., 2015) in which a visible and detectable symptom on a $\mathrm{CH}$ object (referred to as manifestation of damage; e.g. crack, material loss) is induced by a single or a combination of damaging processes (referred to as mechanism; e.g. bending, capillary rise) which are led or performed by one or multiple factors (referred to as agents; e.g. water, temperature).

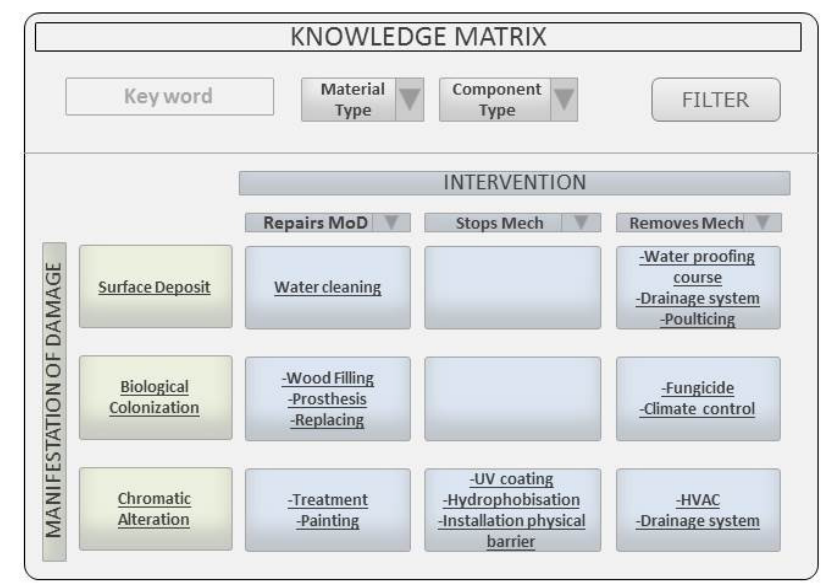

Figure 6. Knowledge matrix

Such understanding of the whole damaging process permits to individuate different typologies of remedial actions, namely: interventions which address (repair) the symptom; interventions which tackle (stop) the damaging process; actions which remove the damaging factor. Based on such principles, the knowledge matrix consists of a matrix-like interface (Fig.6) in which damages are displayed on the vertical axis while cells show the possible interventions, grouped according to their typology. This implies that in each row (that is for each manifestation of damage) three different sets of interventions can be individuated, being in the first column the action repairing the symptom, in the second column the interventions stopping the damaging mechanism and in the third column the actions removing the damaging agents. Interventions provided in the cells of the matrix if clicked are linked to an explanatory card (see Fig.13) which involves: the procedure to carry out the intervention, the materials or components that should be used, its limitations and advantages together with a gallery of relevant images.

\subsection{Terminology editor}

The terminology editor (Fig.7) is a web application designed for browsing or editing the terminology used in all MONDIS software components. The interface, in the read-only mode, enables users to visualize definitions, synonyms, translations 
and images related to a term as well as its position within the related taxonomy. Registered users are allowed to access the full editing version of the terminology editor which permits the following actions: creation of a new term by typing in the top left text field and clicking on the 'create new term' button; insertion of translations and synonyms by adding a new 'label' text field (to add green ' + ' button should be used) and specifying the language code required (e.g. 'en' for English); inputting multiple definitions by employing the 'definition' text field; providing comments to the term in the 'comments' text field including relevant notes to better understand a concept; defining 'broader terms' which means positioning the term within a taxonomy by specifying the immediate broader term available in the ontology to which it belongs (e.g. 'wood' is assigned to broader term 'organic natural materials'); uploading pictures related to the term; providing 'tags' as an additional reference for exploiting the term in a customized group of concepts. Data inserted in the terminology editor are provided with a 'source link' in order to assign the reference from which the information is taken (e.g. books, papers, phot archive etc.).

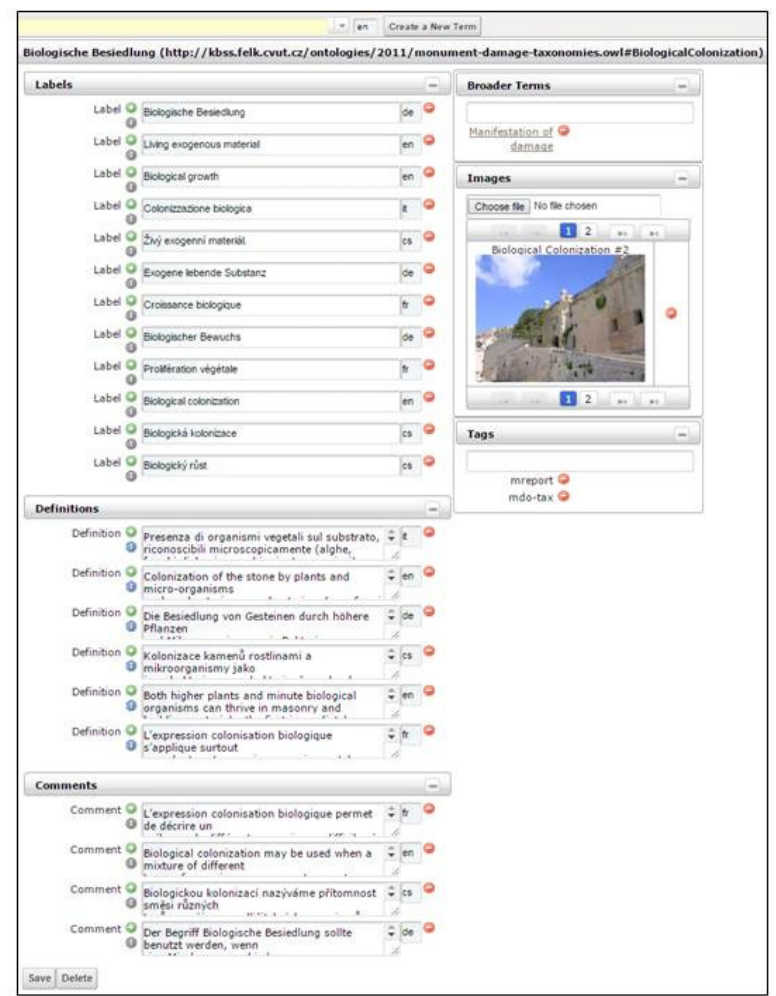

Figure 7. Terminology editor

\section{RECORD EXAMPLE}
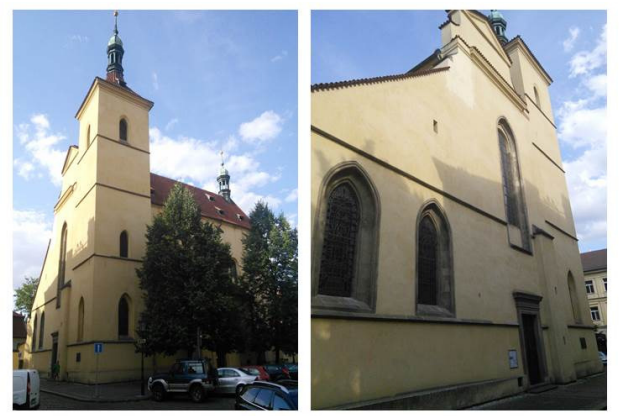

Figure 8. St. Haštal church
The church of Saint Haštal (Fig. 8) in the Old Town, Prague is one of the most significant ecclesiastical monuments in the city. Dating back to the $10^{\text {th }}$ century and rebuilt in the second half of the $14^{\text {th }}$ century, the building represents a great example of Gothic architecture in the region.

For the purpose of presenting MONDIS tools in a straightforward and understandable manner, a simple case is here discussed: a crack on the external wall of the building is reported (section 3.1), additional information concerning the structural evolution and the surrounding characteristics are provided (section 3.2) and finally possible remedies are retrieved from the system (section 3.3).

\subsection{On-site inspection data}

Information related to damage identification has been gathered directly on-site using the MONDIS mobile application. The input is as following:

1) Building identification- name/denomination: Saint Haštal; morphological type: hall/church; structural type: load bearing wall; functional type: church; architectonic style: Gothic.

2) Building localization- GPS coordinates automatically uploaded (50.091324763416424, 14.424317765359598)

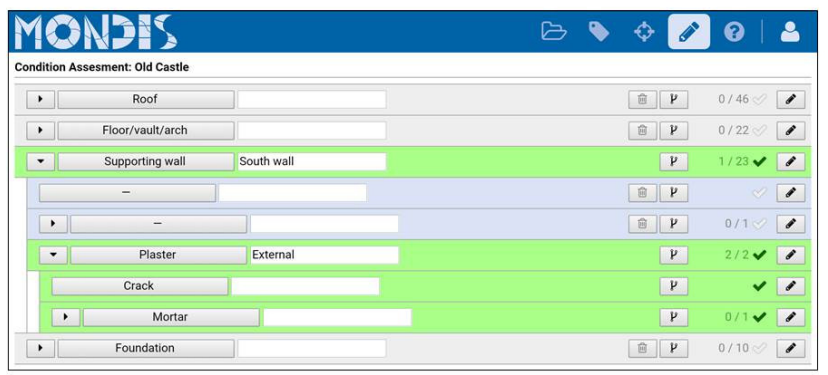

Figure 9. Building components, material and damage

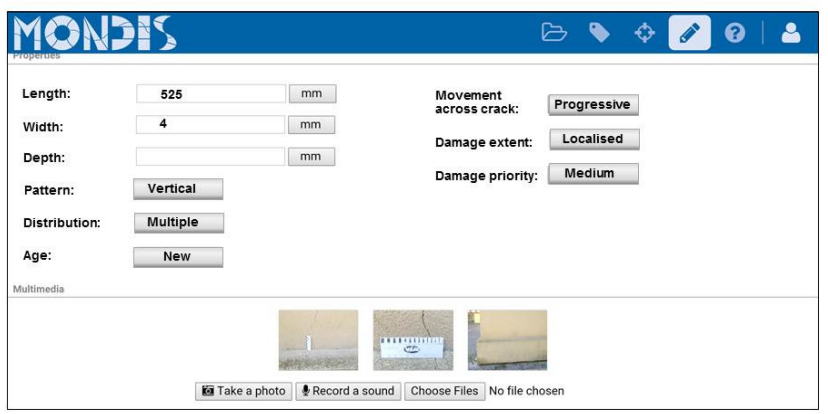

Figure 10. Crack detail assessment

3) Building inspection (Fig.9)- the building component 'supporting wall' is selected with label 'south wall' in order to specify which element the inspection refers to. The subcomponent 'plaster' is selected with label 'external' to properly locate the specific area. To this building component material (mortar) and damage (crack) are assigned. It is important to note that whenever a node in the tree structure is activated it becomes of light blue color and if relevant parameters are measured in the detail assessment screen the row turns green (with check icon).

4) Crack detail assessment (Fig.10)- parameters shown in this screen are context-specific and vary depending on damage, material or component types which are being assessed. In this 
example quantitative and qualitative unknowns for the crack damage are defined- crack length: $525 \mathrm{~mm}$; crack width: $4 \mathrm{~mm}$; crack depth: N/A; pattern: vertical; distribution: multiple; age: new; movement across crack: progressive; damage extent: localized; damage priority: medium. Furthermore a gallery of three photographs, which were taken during the inspection using the 'take a photo' function, is shown.

Once completed, the report is synchronized with the server and data are then validated. Finally the record is published in the MONDIS explorer and available to users of the system.

\subsection{Integration of additional information}

The integration of data related to the structural evolution and the surrounding characteristics of the building under analysis is carried out by means of Ontomind profiles. Firstly, the Saint Haštal record is retrieved and visualized in the MONDIS explorer and the information is added to the entry by selecting the required profile (in this example two profiles are needed, namely the object evolution and the location characteristics profiles) and by clicking on the 'add new profile' button shown at the bottom right corner of the MONDIS explorer, in Figure 4.

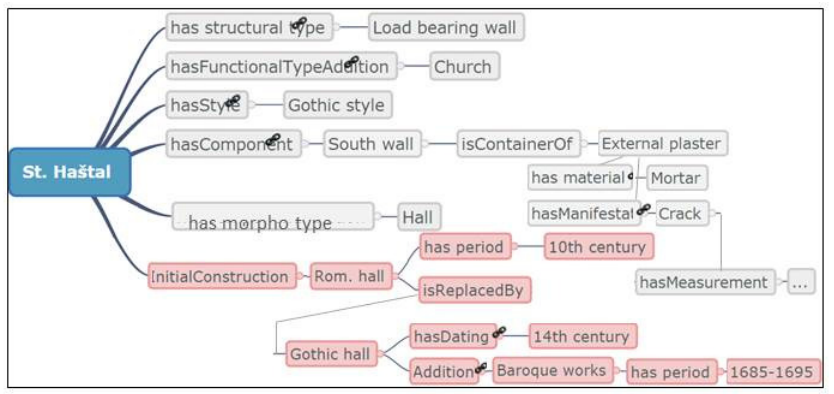

Figure 11. Object evolution profile

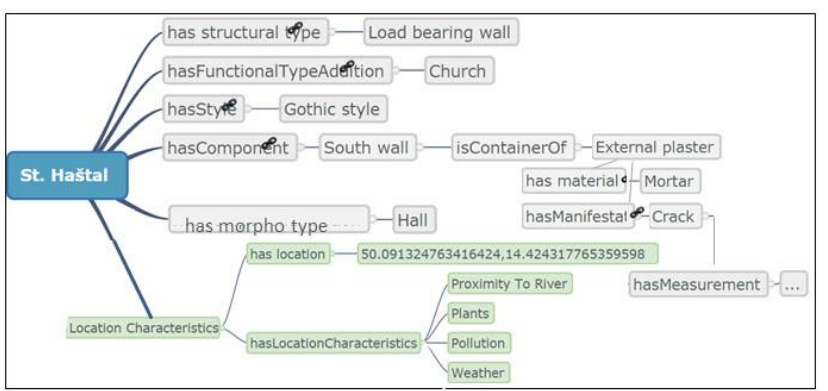

Figure 12. Location characteristics profile

The following data are provided:

1) Object evolution (Fig.11)- the ontological mapping of the data collected via the MONDIS mobile is shown in grey color. The red boxes in the tree-like structure model the object evolution by the available profile-specific semantic links. In particular the profile describes that: Romanesque church built in the $10^{\text {th }}$ century is replaced by the Gothic church founded in the $14^{\text {th }}$ century which in turn experienced some additions and reconstruction works in Baroque style during the period 16851695.

2) Location characteristics (Fig.12)- similarly to the previous profile, data from the on-site inspection carried out with the MONDIS mobile are shown in grey color. Information concerning the surrounding characteristics is provided in green boxes: at the site it is revealed a moderate flood hazard due to the proximity of the church to the river; other relevant threats include the presence of nearby plants, pollution due to local traffic and the adverse impact of climatic conditions, especially in terms of temperature fluctuations and water sources.

Once compiled, the profiles are saved, attached to the record and shown in the MONDIS explorer in the 'other data' section in Figure 4.

\subsection{Searching for possible remedies}

As mentioned in section 2.2, damages reported in a record are semantically linked to possible damaging processes and consequently to feasible interventions. Such information is provided and visualized in the knowledge matrix.

In this example the crack in the plaster has not been diagnosed by the user (to do so a dedicated Ontomind profile must be filled in), therefore the matrix retrieves from all other records stored in the system all possible actions aimed at repairing the crack, stopping the ongoing mechanisms producing the crack as well as at removing the agents performing these damaging process. The search can be further refined by filtering mechanisms and agents which however requires a professional understanding of the root cause of the problem in order to find an optimal intervention.

The interventions suggested by the knowledge matrix include, among others: repointing/partial rebuilding (repair); stitching (stop mechanism; hydrophobization/drainage system/ application of a sacrificial layer (remove agent). Each remedial action can be selected by the user and a detailed intervention card is shown (Fig.13). This card provides general information on how and when to perform the intervention.

Intervention Name: Hydrophobization

Intervention Definition: Hydrophobization is used to protect building structures which are susceptible to the periodic action of water coming from atmospheric precipitation, rainfall and snowfall

\section{Material: Silicone: Wax \\ hydrophobizing agent:}

Organsilicone: Soap:

\section{Component:}

Procedure:Following conditions should be fulfilled: -isolating the pump. When the appropriate amount of vacuum is accumulated at the bottom part of the object, the hydrophobizing solution is introduced by means of a polyurethane hose until the object is saturated. The implementation of vacuum impregnation of the facade is quite complicated as it requires the object to be tightly sealed in order to produce a void. The ground contacting the masonry needs to be impregnated with the solution and subsequently

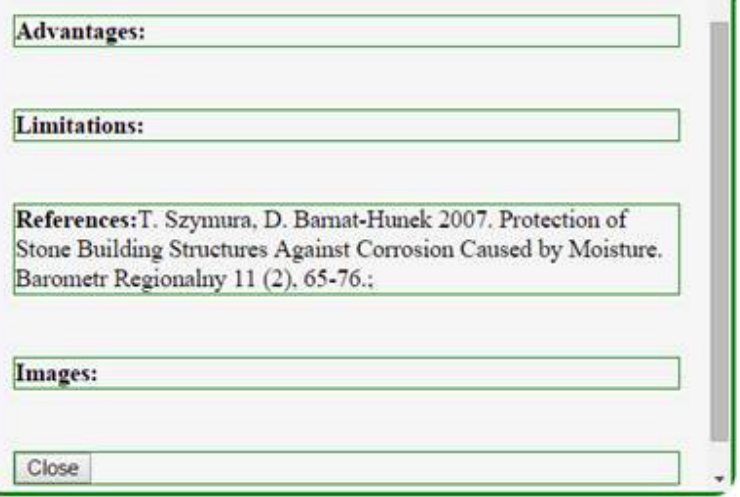

Figure 13. Intervention detail card 


\section{CONCLUSIONS}

MONDIS attempts to fill the research gaps in ontology engineering and data management systems, in the context of the application of Semantic Web technologies to the field of cultural heritage protection. The main project results include: the formalization of the Monument Damage Ontology which represents the reference platform for the knowledge-based tools, providing for semantic searching and automatic reasoning; the inputting (MONDIS mobile/desktop and Ontomind profile) and visualization (MONDIS explorer, the knowledge matrix and the terminology editor) applications intended to support users into performing different conservation tasks such as building inspection, data digitalization and knowledge retrieval. Tools are accessible via the portal www.mondis.cz.

The advantages of the MONDIS tools rely on the flexibility and adaptability of the ontology to provide for semantic autocompletion, knowledge and association discovery, and advanced semantic visualizations; furthermore the compatibility of software components allow their integration in a userfriendly, comprehensive information system which enable users to gather, share and process data in the same working environment. Nevertheless some limitations should be underlined: the ontological representation presents, due to modelling requirements, a certain level of 'artificiality' which derives from a subjective understanding of the domain; the knowledge-based tools are intended for highly specialized conservation tasks, limiting the accessibility to a wider nonexpert user base.

The envisaged future developments might include formalizing high-granularity knowledge in specific areas of the domain, strengthening community building activities for the population and sustainability of the system and implementing the project results into policy strategies especially at a local and regional level.

\section{ACKNOWLEDGEMENTS}

This work has been supported by the grant No. DF11P01OVV002 "Defects in immovable cultural heritage objects: a knowledge-based system for analysis, intervention planning and prevention" of the Ministry of Culture of the Czech Republic.

\section{REFERENCES}

Blaško, M., Cacciotti R., Křemen P., Kouba Z., 2012. Monument Damage Ontology. Lecture Notes in Computer Science (7616), pp. 221-230.

Cacciotti, R., Blaško, M., Valach, J., 2015. A diagnostic ontological model for damages to historical constructions. Journal of Cultural Heritage, 16 (1), pp. 40-48.

Cacciotti, R., Valach, J., Kuneš, P., Čerňanský, M., Blaško, M., Křemen, P., 2013. MONument Damage Information System (MONDIS): an ontological approach to cultural heritage documentation. ISPRS Annals of the Photogrammetry, Remote Sensing and Spatial Information Sciences, II-5/W1, p.55-60.
Doerr, M., 2003. The CIDOC CRM - an ontological approach to semantic interoperability of metadata. AI Magazine, 24(3), pp. 75-92.

EC DG Information Society, 2002. Full report "The DigiCULT Report- Technological Landscapes for Tomorrow's Cultural Economy Unlocking the Value of Cultural Heritage", Brussels, Belgium ftp://ftp.cordis.lu/pub/ist/docs/digicult/full_report.pdf.

European Commission, 2006. Booklet "On the horizon: preserving digital information with CASPAR", http://cordis.europa.eu/docs/projects/cnect/2/033572/080/publis hing/readmore/CASPAR-booklet.pdf.

European Communities, 2009. Booklet "CHARISMA: Cultural Heritage Advanced Research Infrastructures: Synergy for a Multidisciplinary Approach to Conservation/Restoration", https://ec.europa.eu/research/infrastructures/pdf/charisma.pdf.

Faletar, S., 2005. Short overview of a pan-European cultural heritage project, CALIMERA. Library Hi Tech News, 22(2), pp. 23-24.

Konstantinos, N., Vavliakis, G., Karagiannis, Th., Mitkas, P., 2012. Semantic web in cultural heritage after 2020. In: 11th International Semantic Web Conference 2012 (ISWC 2012), Boston, USA, pp. 1-6.

Jokilehto, J., 1990. Report by ICCROM Working Group 'Heritage and Society' "Definition of cultural heritage", Rome, Italy,http://cif.icomos.org/pdf_docs/Documents\%20on\%20line/ Heritage\%20definitions.pdf.

Meghini, C., Risse, T., 2005. BRICKS: A digital library management system for cultural heritage. ERCIM News, (61), pp. 54-55. 